\title{
Triticum aestivum ssp. vulgare and ssp. spelta cultivars. 1. Functional evaluation
}

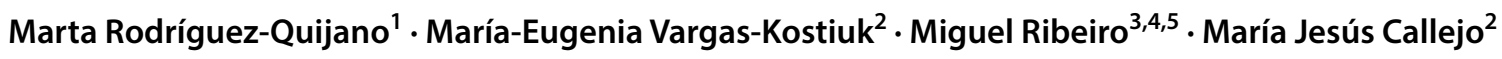

Received: 7 November 2018 / Revised: 29 January 2019 / Accepted: 24 February 2019 / Published online: 1 March 2019

(c) Springer-Verlag GmbH Germany, part of Springer Nature 2019

\begin{abstract}
Over recent decades, several types of ancient grain varieties have been reintroduced through organic farming systems. In the past 20 years, there has been a renewed interest in growing spelt, and this species has a market niche in Europe and North America in natural, organic and healthy products. Are there any differences between commercial wheat varieties and spelt? The analysis of the bread-making quality of three bread wheat and two spelt cultivars has found no significant differences between the two types of wheat in protein, $\mathrm{Fe}$ or $\mathrm{Zn}$, although differences were observed between cultivars. In mean quality parameters, a marked difference was observed between the bread wheat and spelt cultivars, the latter having poorer alveo-consistographic characteristics, indicative of weaker gluten and dough. With the exception of the variety 'Craklin', the gliadin/glutenin (Gli/Glu) ratio is negatively correlated with the technological quality of cultivars. Principal component analysis shows that the two commercial wheats 'Bonpain' and 'Sensas' lying close to gluten strength parameters; and, dough extensibility was associated with total gliadin content and the Gli/Glu ratio, both types of spelt being more closely related to these parameters, indicating that spelts are associated with greater dough extensibility.
\end{abstract}

Keywords Bread wheat $\cdot$ Spelt wheat $\cdot$ Quality wheat $\cdot$ RP-HPLC $\cdot$ Organic farming

\section{Introduction}

Spelt (Triticum aestivum ssp. spelta L., $2 n=6 x=42$, genome AABBDD) is an ancient type of hulled wheat and was the main grain used for making bread in Europe from the fifth to the early twentieth century [1]. Due to advances in bread wheat (Triticum aestivum ssp. vulgare L., $2 n=6 x=42$,

Marta Rodríguez-Quijano

marta.rurquiaga@upm.es

1 Unit of Genetics, Department of Biotechnology, Plant Biology, UPM, Ciudad Universitaria, 28040 Madrid, Spain

2 Department of Chemistry and Food Technology, UPM, Ciudad Universitaria, 28040 Madrid, Spain

3 CQ-VR, Chemistry Research Centre, Chemistry Department, University of Trás-os-Montes and Alto Douro, 5000-801 Vila Real, Portugal

4 Department of Genetics and Biotechnology, University of Trás-os-Montes and Alto Douro, 5000-801 Vila Real, Portugal

5 Functional Genomics and Proteomics Unit, University of Trás-os-Montes and Alto Douro, 5000-801 Vila Real, Portugal genome AABBDD) and the introduction of maize in Europe, spelt stopped being grown in many places. Currently, it is only grown in some areas in North America and in Europe, where it can be found in some isolated regions such as in Asturias (North of Spain) and some regions of Germany and Switzerland. Modern varieties of bread wheat have higher yields, lower production costs and better bread-making characteristics.

Nevertheless, over recent decades, several types of ancient wheat varieties have been reintroduced. In particular, there has been a renewed interest in growing spelt, and this species has a market niche in organic and healthy products. This is attributable to various factors: the fact it is a hulled wheat (i.e. the grains are not readily separated from the glumes) means that spelt is more resistant to pathogens and adverse conditions, making it particularly suitable for organic farming [2]. Further, in some genotypes of spelt, high concentrations of zinc, iron and protein have been detected, being highly stable under different environmental conditions [3] .In addition, spelts have a higher lipid content and lower insoluble and total fibre content than wheat [4].

In wheat flours, proteins contribute to the three main quality attributes that determine bread-making potential: 
protein content of the flour, rheological properties of the dough and grain hardness. The first two of these parameters relate to the importance of the quantity and quality of protein, while the third, hardness, is related to the interaction (or lack of thereof) between proteins surrounding the starch granules and the protein matrix of the endosperm.

Since the nineteen-seventies, research on dough quality has mainly focused on assessing the composition of wheat grain proteins and especially of the proteins in flour that form gluten. The proteins that form the viscoelastic complex known as gluten are prolamins: glutenins and gliadins. Glutenins, polymeric proteins, can be classified by their molecular weight, assessed using sodium dodecyl sulfate polyacrylamide gel electrophoresis (SDS-PAGE), into high and low molecular weight glutenin subunits (HMW-GS and LMW-GS, respectively). It is considered that HMW-GS are the gluten proteins with the greatest influence on the rheological properties of flour and are encoded by Glu- 1 loci, on the long arm of the homoeologus group 1 chromosomes [5].On the other hand, gliadins are monomeric proteins and can be classified into $\omega 5-, \omega 1.2-, \alpha / \beta$ - and $\gamma$-gliadins on the basis of their primary structure and molecular weight, using reversed-phase high-performance liquid chromatography [6].

In allohexaploid wheat, grain hardness (or endosperm texture) is an important quality factor and, for this reason, wheat is generally traded by and classified into 'hard' and 'soft' classes being hardness related with puroindoline A and B proteins [7]. The genes that code for these proteins, Pina-D1 and Pinb-D1, respectively, have been localised on the short arm of the 5D chromosome (the hardness $[\mathrm{Ha}]$ locus) [8]. When both genes are in their wild type, the grain texture is soft, while mutations in either of the genes result in a hard grain texture [9].

The objective of this study was to compare the flour and bread-making potential of bread wheat against spelt wheat. Three bread wheat cultivars and two spelt wheat cultivars were selected for this study. To minimize the environment influence, all the samples were grown in the same year under the same soil and climate conditions.

\section{Materials and methods}

\section{Plant materials}

Three cultivars of bread wheat, 'Bonpain', 'Craklin' and 'Sensas', and two local cultivars of spelt wheat, 'Espelta Álava' and 'Espelta Navarra', were used for the present study. $25 \mathrm{~kg}$ of grain of each variety, grown organically in Vitoria (Álava, Spain) $\left(42^{\circ} 51 \times 2^{\prime \prime} \mathrm{N}, 2^{\circ} 40 \times 21^{\prime \prime} \mathrm{W}\right)$ in 2010/2011, were kindly provided by the Diputación Foral de Álava (Spain).

\section{Grain protein composition}

Gluten proteins were extracted from wheats using a sequential procedure [10]. HMW-GS were separated by SDS-PAGE according to [11]. HMW-GS composition was identified using the nomenclature of [12]. Discrimination between HMW-GS $2 *$ and $2 \bullet \bullet$ subunits the Glu-Al locus and between the 7 and $7 *$ subunits of the Glu-Bl locus was based on PCR reactions with the specific primers as [13] and [14], respectively. A similar approach was used to identify the puroindolin alleles present in the varieties at the PinaD1 and Pinb-D1 loci [15]. DNA was extracted following a standard CTAB protocol [16].

For quantification of glutenin and gliadin subfractions, prolamins were separated on the basis of hydrophobicity by reversed phase-high performance liquid chromatography (RP-HPLC) as described [17].

\section{Quality analyses}

The 1000 grain weight was determined by counting 1000 grains using a seed counter (Pfeuffer, Mess-und Prüfgeräte) and weighing them with digital scale. Grain hardness, protein, Fe and $\mathrm{Zn}$ content, and sodium dodecyl sulphate sedimentation volume (SDSS test) were determined using wholemeal flours, obtained with a Tecator mill with a 1-mm screen. The flours for measuring rheological properties, with an alveograph and consistograph, were milled using a Ceramic Heger GmbH and Co. HG, Kv10 mill. After milling with the same procedure, the fractions extracted from each one of the five cultivars were mixed for $20 \mathrm{~min}$ in a flour blender (Chopin Technologies) at $48 \mathrm{rpm}$.

Kernel hardness was estimated by near-infrared reflectance (NIR) using a PerCon Inframatic 8120 spectrometer (Perten Instruments GmbH, Germany), according to Method 39-70.02 [18]. Whole protein content was measured by NIR using a Technicon Infralyzer 300 spectrometer (Technicon Instrument Co. Ltd, Hants, U.K.).

To assess mineral iron (Fe) and zinc ( $\mathrm{Zn})$ content the [19] method was followed. Gluten strength was estimated with an SDSS test [20].

Viscoelastic behaviour of samples was determined using an NG-97 Alveograph (Chopin Technologies, Tripette et Renaud, France) (Method 54-30A.2010 [18]). The effect of wheat flour on dough rheology during mixing was determined using a Consistograph (Chopin Technologies, Tripette et Renaud, France) (Method 54-50.2000 [18]. All measurements were performed in two replications, except the SDSS test, in four replications. 


\section{Data analysis}

Statistical analysis was performed using SAS statistical software [21]. Differences between the different wheats were assessed by one-way analysis of variance (ANOVA). Multiple comparisons were made using Tukey's post hoc test (significance $p<0.05$ ). Pearson's correlation coefficients were calculated using the CORR procedure. Principal component analysis was applied for multivariate statistical analysis using InfoStat software [22].

\section{Results and discussion}

\section{Characterization of HMW-GS and puroindolines}

To assess heterogeneity, 100 randomly selected grains of each cultivar were analysed using SDS-PAGE. Table 1 lists the HMW-GS and puroindoline composition for each cultivar. In the case of 'Espelta Álava', we found different prolamin profiles, indicating that it is a heterogeneous cultivar (as is the case in the landrace of wheat), the other four cultivars having homogeneous electrophoretic profiles.

The varieties under study showed clearly distinct HMW glutenin profiles. The first studies that related the presence of specific HMW-GS with bread-making quality [23] identified HMW-GS 1 (observed in 'Sensas', 'Espelta Navarra' and the heterogeneous spelt variety 'Espelta Álava') and $2^{*}$ (observed in 'Bonpain'), coded by the Glu-Al locus, as being associated with high dough strength and hence "good" bread-making quality, while the null subunit (observed in 'Craklin') is related to "poor" bread-making quality. There is much less studies about the influence of HMW-GS $2 \bullet \bullet$ (observed in 'Espelta Álava') on dough strength but a beneficial effect similar to that of subunit $2^{*}$ has been reported for this subunit in a double haploid wheat population [24] and in Spanish bread wheat landraces [13].

The subunit pairs coded by the Glu-Bl locus found in the three bread wheat varieties $\left(7+8,7^{*}+9\right.$ and $\left.17+18\right)$ have already been described in commercial bread wheat, all being related to high dough strength [25]. In 'Espelta Álava', we found subunit pairs $13+16$ and $13+18$. The latter has only been described previously in another spelt cultivar of Spanish origin [26] and its influence on quality remains unknown. In the review of [27], the subunit pair $13+16$ was described as having a good influence on bread-making quality.

The subunit pair $6 *+8^{*}$ found in 'Espelta Navara' was identified by [28] in Canadian bread wheats. This pair has not been described in Spanish [26] or Central European [29, 30] spelt varieties although it has been observed analysing the HMW-GS of the 'Obelkurmer' and 'Bättig Rothen' Central European spelt varieties and as in 'Dawbull' (the standard for this subunit pair) (unpublished data).

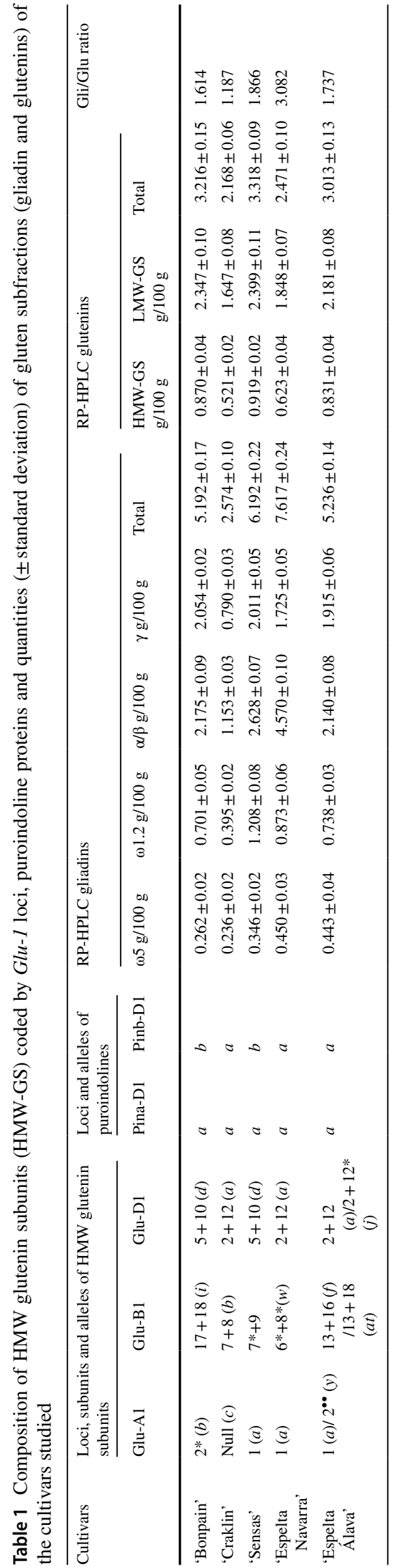


The Glu-Dl locus codes for the HMW-GS pairs $5+10$ in the 'Bonpain' and 'Sensas' and the pair $2+12$ in 'Craklin' and in the two spelt varieties 'Espelta Navarra' and 'Espelta Álava'. Some grains of this latter cultivar presented the subunit pair $2+12 *$, considered rare though described in Italian cultivars [31] and in Spanish landraces of common and spelt wheat (e. g., [26]). Based on SDSS test, its influence on dough strength seems similar to that of the subunit pair $2+12$ (Dr Rodriguez-Quijano, personal communication), that is, "poor" compared to the allelic pair $5+10[25,27]$.

Considering puroindoline allelic composition (Table 1), varieties'Bonpain' and'Sensas' have moderate endosperm hardness while 'Craklin' and the two spelts, having the wildtype $a$ allele at both puroindoline loci, have a low endosperm hardness [32].

\section{Quantification of prolamin subfractions}

To complement the electrophoresis used to identify different prolamins proteins (HMW-GS, LMW-GS and gliadins), analysis based on RP-HPLC enabled us to perform quantitative analysis of these proteins [6] and the results are summarized in Table 1.

Regarding gliadins, either total gliadin and the proportions of the different gliadin types varied considerably among the wheat cultivars analysed, being this diversity in agreement with previous studies $[6,15]$.Within the gliadin types (Table 1 ), the $\alpha / \beta$-type gliadins were generally the most abundant, followed by the $\gamma$-type gliadins. The $\omega$-type gliadins were present at lower levels but, among them, the $\omega 1.2$-type was dominant. The highest levels of $\omega 5$-gliadins and $\omega 1.2$-gliadins were found in the two spelt varieties and in 'Sensas'. The highest levels of $\alpha / \beta$-gliadins were observed in 'Espelta Navarra' followed by 'Sensas'. With regard to $\gamma$-gliadins, 'Bonpain' and 'Sensas' showed the highest levels, followed by 'Espelta Álava,' whereas 'Craklin' had the lowest levels of all gliadin fractions. Notably, $\gamma$-type gliadins have been shown to be positively correlated with dough strength [33] and also with loaf volume, suggesting a positive contribution to bread-making [34]. The $\omega 1.2$-type produces the least positive effects on mixing tolerance and loaf volume [35], suggesting that it is less important for breadmaking than the other gliadin types.

The lowest levels of HMW- and LMW-GS were found in 'Espelta Navarra' and 'Craklin'. In the case of 'Espelta Navarra' it was associated with the highest Gli/Glu ratio, a parameter that has been inversely related with good dough strength properties. If 'Craklin' (in which the low protein content could have influenced the analysis) is excluded, the Gli/Glu ratio would predict the following bread-making quality: 'Bonpain' > 'Espelta Álava' > 'Sensas' > 'Espelta Navarra'.
Several other authors have described some key differences between spelt and bread wheats in terms of the quantity of the different glutenin and gliadin fractions (see [36]). Spelt is characterized by a much higher total gliadin and lower total glutenin content. The Gli/Glu ratio is significantly higher in spelt, and $\alpha / \beta$-gliadins are predominant, followed by $\gamma$-gliadins while LMW-GS, $\omega$-gliadins and HMW-GS are generally minor components [37]. Noteworthily, when the cultivars are individually considered, the amounts of all subfractions in 'Espelta Álava' are more similar to the values in bread wheat 'Bonpain' and 'Sensas' than to those found in 'Espelta Navarra'.

\section{Cultivar quality}

About grain quality, in all cases, the ANOVA tests conducted have demonstrated a highly significant effect of the cultivar in the variation found for grain quality as shown in Table 2. In this sense, Table 3 shows the mean values of grain hardness, rheological parameters and others related to the nutritional value, such as the $\mathrm{Fe}$ and $\mathrm{Zn}$ content, found for each of the cultivars studied.

'Bonpain' and'Sensas' bread wheat varieties had higher endosperm hardness than the spelt and 'Craklin' varieties. This result is the expected according to the allelic composition observed for puroindoline loci (Table 1). Among the three soft endosperm cultivars, the spelt varieties had significantly higher hardness values than 'Craklin'. This may be related to the low protein content in 'Craklin' (Table 3) since a positive correlation has been found between grain hardness and protein content [38].

The nutritional value of a wheat variety is largely dependent on the grain protein, $\mathrm{Fe}$ and $\mathrm{Zn}$ content. Protein content is known to be very sensitive to environmental conditions and has a very low heritability. Some authors [2] found a high protein content in spelt genotypes; however, the two spelt varieties had protein content values in the middle of the overall range for the five cultivars analysed (Table 3). Fe and $\mathrm{Zn}$ content varied significantly between cultivars but again the kind of wheat cultivar, either common or spelt, was not a defining criterion. So 'Espelta Navarra' had the highest content of both micronutrient elements among all varieties under study while 'Espelta Álava' showed the lowest mean values.

\section{Flour and bread-making quality}

According to the genetically determined quality, bread-making quality can be expected to differ between the cultivars analysed. The ANOVA tests confirmed the higly significant influence of the variety in the variation found for all parameters (Table 2). 
Tukey's test revealed significant differences in SDSS test means across the five cultivars. The largest mean was recorded for 'Bonpain' and 'Sensas', which agrees with the presence of the beneficial HMW-GS pair $5+10$ coded by the Glu-Dl locus, although 'Bonpain' had a significantly larger SDSS test than 'Sensas'; considering the HMW-GS composition of these cultivars, the difference might be due to the pair $17+18$ [39]. The two spelt varieties had intermediate SDSS test though 'Espelta Álava' was significantly higher than 'Espelta Navarra'; considering that the main difference in HMW-GS composition between these varieties lies in the Glu-B1 locus, a more positive effect of the pair $13+16$ over the pair $6^{*}+8^{*}$ can be suggested. These results can also be partly explained by the quantity of glutenins and gliadins and particularly the (Gli/Glu) ratio (Table 1). 'Bonpain' and 'Sensas' showed indeed very similar amounts of glutenins, but 'Sensas' had a higher Gli/Glu ratio, known to negatively affect gluten properties. Similarly, 'Espelta Navarra' showed a markedly higher Gli/Glu ratio than 'Espelta Álava'. The smallest SDSS test for 'Craklin' is likely due to a prolamin profile associated with low gluten strength at both Glu-Al and Glu-Dl loci. Some negative influence of the low protein content in this variety, and specifically of the very low amount of $\gamma$-gliadins, must also be taken into account.

With regard to the alveograph parameters (Table 3), 'Bonpain' and 'Sensas' had significantly higher mean $W$ values than either of the spelt varieties and 'Craklin', while the highest $W^{\prime}$ values were found for 'Bonpain' and the lowest for the two spelt varieties and 'Craklin'. Regarding tenacity, 'Sensas' had a significantly higher $P$ value than other varieties, followed by 'Bonpain', and differences between the two spelt varieties were not significant. Taking into consideration that 'Espelta Álava' had significantly higher L than 'Espelta Navarra', no difference was observed between 'Bonpain' and both spelt cultivars. Analysing the alveograph curve, the two spelt varieties obtained the lowest mean $\mathrm{P} / \mathrm{L}$ ratios.

Consistographic parameters could not be measured for the 'Craklin' cultivar at constant hydration as the dough did not reach a consistency equivalent to the target pressure of $2200 \mathrm{mb}$. Analysing the consistograms (Table 3) for the other cultivars, the dough behaviour differed between the commercial wheats and the spelt varieties. The spelts obtained lower mean values for HYD, TprMax and Tol and higher mean values for dough weakening $\left(D_{250}\right.$ and $\left.D_{450}\right)$, although the mean $D_{450}$ of 'Bonpain' was not significantly different to those of the two spelt varieties.

The alveo-consistographic analyses indicate that the wheats with the best rheological properties were 'Bonpain' and 'Sensas'. This finding is related to their greater endosperm hardness, and stronger gluten, which is indicated by the significantly higher values for most parameters associated with good bread-making behaviour (i. e., SDSS test, $W$, $W^{\prime}$, HYD, TrpMax and Tol; see Table 3). Filipče et al. [40] 


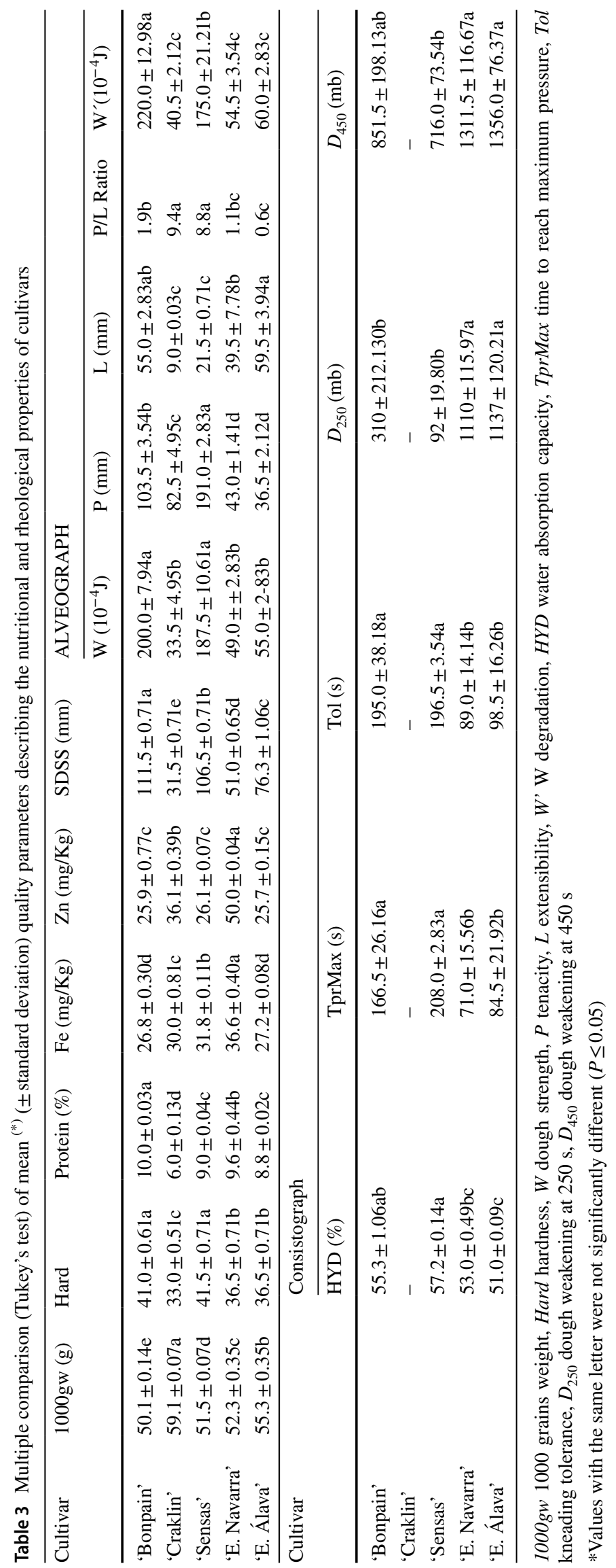




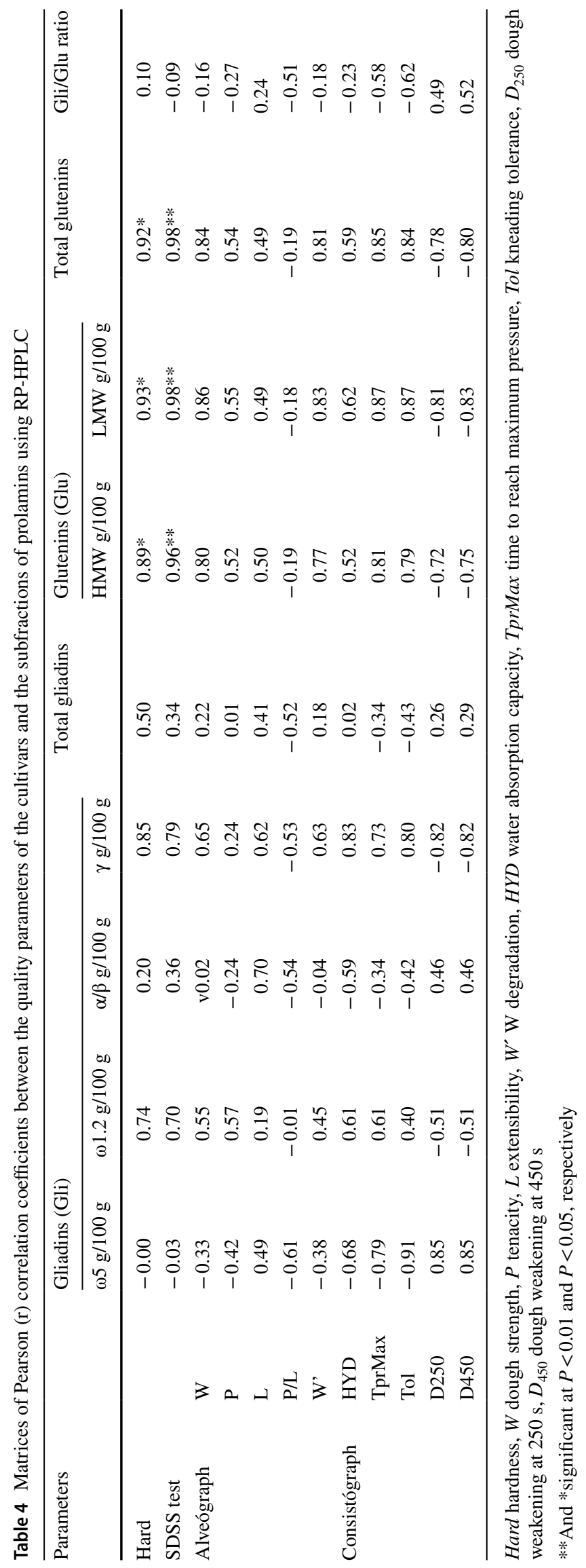


Fig. 1 Representation of the variables and the samples on the first and second principal component axes. $W$ strength, $P$ tenacity, $L$ extensibility, $P / L$ tenacity/extensibility ratio, Totalglu Total glutenis, Totalgli Total gliadins, Gli/Glu gliadin/ glutenin ratio

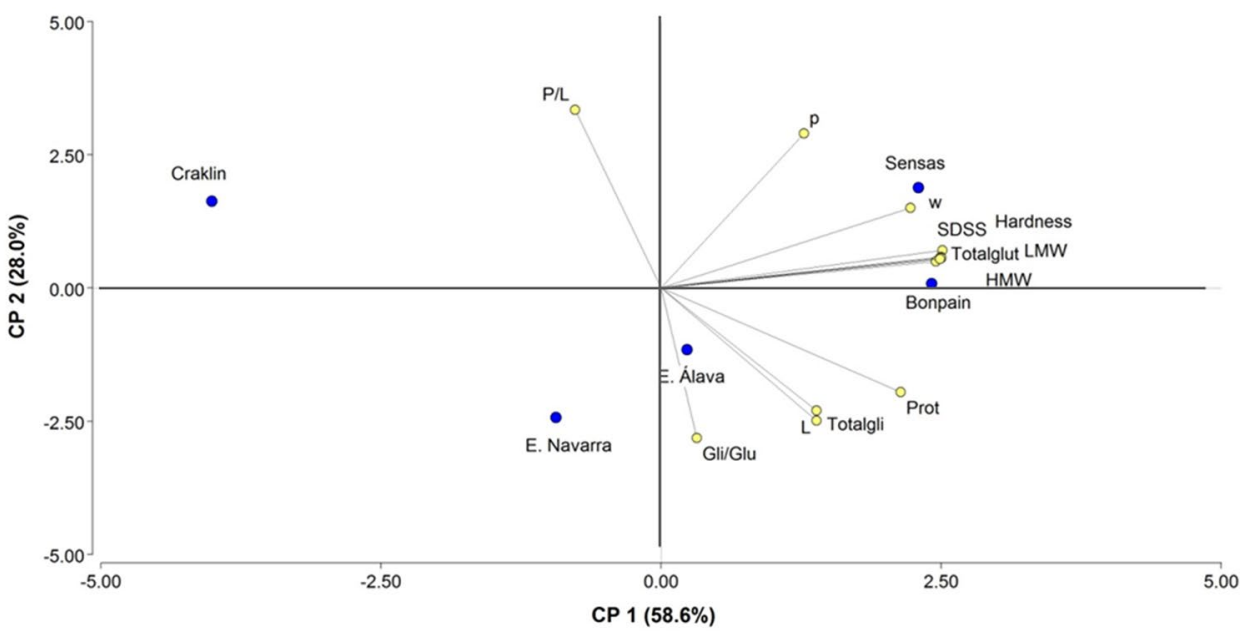

analysed three spelt genotypes by a Brabender farinograph and found the doughs to have poor to moderate rheological properties and poor stability. In our study, we found lower TprMax for spelt doughs, this also indicating a weak gluten.

McRitchie [41] reported that the addition of gliadins shortened mixing time and reduced dough stability, and other studies have found the quantity of HMW- and LMWGS to be correlated positively with bread-making quality and negatively with the Gli/Glu ratio [42].

Table 4 shows the coefficients for the correlation between the quantity of gliadin and glutenin fractions and the Gli/ Glu ratio in the cultivar analysed and the quality parameters. The quantities of $\omega 1.2-$ and $\gamma$-gliadins showed positive but non-significant correlations with endosperm hardness, strength parameters (SDSS test, $W$ and $W^{\prime}$ ), coinciding with [33] and the consistograph parameters HYD, TprMax and Tol. Furthermore, it is to be noted that $D_{250}$ and $D_{450}$ were negatively correlated with the quantities of these gliadins and positively correlated with the quantities of $\omega 5$-gliadins. Extensibility (L) was positively correlated with all gliadin fractions though the correlation coefficients were not significant. We have found significant positive correlations between the quantities of glutenins (HMW-GS, LMW-GS and total) and endosperm hardness and SDSS test. Strong but non-significantly positive correlations were found with strength parameters ( $W$ and $W^{\prime}$ ) and consistographic parameters (TprMax and Tol), while the correlation with $D_{250}$ and $D_{450}$ was negative but not significant. Correlations between most quality parameters and the Gli/Glu ratio were negative.

Pruska-Kedzior et al. [37] indicated that the rheological properties of spelt gluten are influenced by gliadins that act as a viscous monomeric plasticiser, while those of gluten in bread wheat varieties are influenced by the glutenins that act as polymeric factor promoting networking. In our study, 'Espelta Álava', with the typical genetic characteristics of an
Asturian spelt, is notably different from 'Espelta Navarra', that is more typical of a Central European origin. Regarding gliadin and glutenin subfraction composition 'Espelta Navarra' was found to be more similar to the commercial varieties analysed in our study. That is, although our results do indicate differences in rheological properties between spelt and bread wheat varieties, we also found differences between the spelts themselves (protein content and SDSS test).

In order to extract the major sources of variation in the content of the different gluten proteins and the quality parameters of the flours analysed, a principal component analysis (PCA) was performed (Fig. 1). The PCA score plot of the PC1 versus PC2 explains $86.6 \%$ of the original variation in the data, accounting for $58.6 \%$ and $28.0 \%$ the first and the second components, respectively.

Our findings can be summarised by the principal component analysis (Fig. 1). This type of analysis, based on a correlation matrix, was performed with the values of the glutenin and gliadin fractions and the quality parameters of the flours obtained from the five cultivars. The first two components explain $86.6 \%$ of the variation in the data, the first and second components accounting for $58.6 \%$ and $28.0 \%$, respectively. It can be inferred that these two principal axes account for a high percentage of the total variation, in turn, allowing appropriate interpretation of the data (Fig. 1). The parameters associated with gluten strength (SDSS test, $W$ and hardness) were grouped in the positive part of the first component together with the HMW- and LMW-GS content, the two strong cultivars, 'Sensas' and 'Bonpain', lying close to these parameters. Dough tenacity and the $\mathrm{P} / \mathrm{L}$ ratio, in the positive part of the second component, were negatively correlated with extensibility, total gliadin content and the Gli/Glu ratio, both spelts lying closer to these parameters. Additionally, it can be observed that the cultivar 'Craklin' lies apart from the other cultivars. 


\section{Conclusions}

Differences in rheological properties have been found comparing bread and spelt wheat cultivars. In the alveographic analysis, the flour from two spelt produced dough with lower values for strength, tenacity and degradation, than that from strong bread wheat varieties ('Bonpain' and 'Sensas'), indicating a weaker gluten in these cultivars, though the values were higher than for the bread wheat 'Craklin'.

In addition, the behaviour of the spelt doughs was markedly different from that of dough from 'Bonpain' and 'Sensas' during the kneading process, in terms of hydration, time to reach maximum pressure tolerance to kneading and dough weakening at $250 \mathrm{~s}$ and at $450 \mathrm{~s}$ the behaviour corresponding to doughs with weaker gluten.

In the principal component analysis, the wheat varieties 'Bonpain' and 'Sensas' lie close to gluten strength parameters (SDSS test, strength and hardness) and to HMW- and LMW-GS content, while the two spelts are closer to extensibility, total gliadin content and Gli/Glu ratio, implying that they both yield a more extensible dough.

In general, we can conclude that the differences observed in this study between the cultivars of bread and spelt wheat varieties are not so much due to differences between subspecies as to differences in the genetic composition of cultivars.

Acknowledgements The authors would like to thank the Diputación Foral de Álava for their collaboration with this work. This work was supported by Grant No. AGL 2016-77149 from the Spanish Ministry of Economy and Competitiveness.

\section{Compliance with ethical standards}

Conflict of interest The authors declare that they have no conflict of interest.

Compliance with ethics requirements This article does not contain any studies with human or animal subjects.

\section{References}

1. McFadden ES, Sears ER (1946) The origin of Triticum spelta and its free-threshing hexaploid relatives. J Hered 37:81-89. https:// doi.org/10.1093/oxfordjournals.jhered.a105590

2. Bonafaccia G, Galli V, Francisci R, Mair V, Skrabanja V, Kreft I (2000) Characteristics of spelt wheat products and nutritional value of spelt wheat-based bread. Food Chem 68:437-441

3. Gómez-Becerra HF, Erdem H, Yazici A, Tutus Y, Torun B, Ozturk L, Cakmak I (2010) Grain concentrations of protein and mineral nutrients in a large collection of spelt wheat grown under different environments. J Cereal Sci 52:342-349. https://doi.org/10.1016/j. jcs.2010.05.003

4. Escarnot E, Dornez E, Verspreet J, Agneessens R, Courtin CM (2015) Quantification and visualization of dietary fibre components in spelt and wheat kernels. J Cereal Sci 62:124-133. https ://doi.org/10.1016/j.jcs.2015.01.003
5. Gupta RB, Shepherd KW (1990) Two-step one-dimensional SDSPAGE analysis of LMW subunits of glutelin. Theor Appl Genet 80:65-74

6. Wieser H, Seilmeier W, Belitz HD (1994) Quantitative determination of gliadin subgroups from different wheat cultivars. J Cereal Sci 19:149-155

7. Morris CF (2002) Puroindolines: the molecular genetic basis of wheat grain hardness. Plant Mol Biol 48:633-647

8. Giroux MJ, Morris CF (1997) A glycine to serine change in puroindoline $\mathrm{b}$ is associated with wheat grain hardness and low levels of starch-surface friabilin. Theor Appl Genet 95:857-864

9. Chen F, He Z, Xia X, Xia L, Zhang X, Lillemo M, Morris C (2006) Molecular and biochemical characterization of puroindoline $a$ and $b$ alleles in Chinese landraces and historical cultivars. Theor Appl Genet 112:400-409. https://doi.org/10.1007/s0012 2-005-0095-Z

10. Singh NK, Shepherd KW, Cornish GB (1991) A simplified SDSPAGE procedure for separating LMW subunits of glutenin. $\mathrm{J}$ Cereal Sci 14:203-208

11. Payne PI, Law CN, Mudd EE (1980) Control of homoeologous group I chromosomes of the high-molecular-weight subunit, a major protein of wheat endosperm. Theor Appl Genet 58:113-120

12. McIntosh RA, Yamakazi Y, Dubcovsky J, Rogers WJ, Morris C, Appels R, Xia XC (2013) Catalogue of gene symbols for wheat 2013. In: 12th international wheat genetics symposium 8-13 September 2013, Yokohama, Japan

13. Giraldo P, Rodríguez-Quijano M, Simón C, Vázquez JF, Carrillo JM (2010) Allelic variation in HMW glutenins in Spanish wheat landraces and their relationship with bread quality. Span J Agric Res 8:1012-1023

14. Espí A, Giraldo P, Rodríguez-Quijano M, Carrillo JM (2012) A PCR-based method for discriminating between high molecular weight glutenin subunits $\mathrm{Bx} 7$ and $\mathrm{B} \times 7 *$ in Triticum aestivum L. Plant Breed 131:571-573. https://doi.org/10.111 1/j.1439-0523.2012.01961.x

15. Ribeiro M, Rodríguez-Quijano M, Giraldo P, Pinto L, Vázquez JF, Carrillo JM, Igrejas G (2017) Effect of allelic variation at glutenin and puroindoline loci on bread-making quality: favorable combinations occur in less toxic varieties of wheat for celiac patients. Eur Food Res Technol 243:743-752. https ://doi.org/10.1007/s00217-016-2788-8

16. Saghai-Maroof MA, Soliman KM, Jorgensen RA, Allard RW (1984) Ribosomal DNA spacer-length polymorphisms in barley: mendelian inheritance, chromosomal location, and population dynamics. Proc Natl Acad Sci 81:8014-8018

17. Ribeiro M, Nunes FM, Guedes S, Domingues P, Silva AM, Carrillo JM, Rodríguez-Quijano M, Branlard G, Igrejas G (2015) Efficient chemo-enzymatic gluten detoxification: reducing toxic epitopes for celiac patients improving functional properties. Sci Rep 5:18041. https://doi.org/10.1038/srep18041

18. AACC (2010) Approved methods of the American association of cereal chemists, 11th edn. AACC International, St. Paul, MN, USA

19. Vázquez JF, Chacón EA, Carrillo JM, Benavente E (2018) Grain mineral density of bread and durum wheat landraces from geochemically diverse native soils. Crop Pasture Sci 69:335-346. https://doi.org/10.1071/CP17306

20. Dick JW, Quick JS (1983) A modified screening test for rapid estimation of gluten strength in early-generation durum wheat breeding lines. Cereal Chem 60:315-318

21. SAS Institute Inc (1996) The SAS system for windows. 6.12 edition. SAS Institute Inc. (ed.). Cary

22. Di Rienzo JA, Casanoves F, Balzarini MG, González L, Tablada M, Robledo CW (2011) InfoStat version 2013. Grupo InfoStat, FCA, UN de Córdoba, Argentina 
23. Branlard G, Dardevet M (1985) Diversity of grain protein and bread quality. II. Correlation between high molecular weight subunits of glutenin and flour quality characteristics. J Cereal Sci: 345-354

24. Gobaa S, Kleijer G, Stamp P (2007) 2, a new high molecular weight glutenin subunit coded by Glu-Al: its predicted structure and its impact on bread-making quality. Plant Breed 126:1-4. https://doi.org/10.1111/j.1439-0523.2006.01313.x

25. Cornish BF, Eagles HA, Payne P, Wrigley C, Bushuk W (2006) In: Wrigley C, Békés F, Bushuk W (eds) Gliadin and glutenin. The unique balance of wheat quality. AACC International, St. Paul, Minessota 55121, USA

26. Rodríguez-Quijano M, Vázquez JF, Carrillo JM (1990) Variation of high molecular weight glutenin subunits in Spanish landraces of Triticum aestivum ssp. vulgare and ssp. spelta. J Genet Breed 44:121-126

27. Rasheed A, Xia X, Yan Y, Appels R, Mahmood T, He Z (2014) Wheat seed storage proteins: advances in molecular genetics, diversity and breeding applications. J Cereal Sci 60:11-24. https ://doi.org/10.1016/j.jcs.2014.01.020

28. Ng PKW, Pogna NE, Mellini F, Bushuk W (1989) Glu- 1 allele compositions of the wheat cultivars registered in Canada. J Genet Breed 43:53-59

29. Yan Y, Hsam SLK, Yu JZ, Jiang Y, Ohtsuka I, Zeller FJ (2003) HMW and LMW glutenin alleles among putative tetraploid and hexaploid European spelt wheat (Triticum spelta L.) progenitors. Theor Appl Genet 107:1321-1330. https://doi.org/10.1007/s0012 2-003-1315-Z

30. An X, Li Q, Yan Y, Xiao Y, .Hsam DLK, Zeller FJ (2005) Genetic diversity of European spelt wheat (Triticum aestivum ssp. spelta L.em. Thell.) revealed by glutenin subunit variations at the Glu-1 and Glu-3 loci. Euphytica. https://doi.org/10.1007/s1068 1-005-9002-6

31. Pogna NE, Mellini F, Beretta AM, Dal Belin Peruffo A (1989) The HMW-GS of common wheat cultivars grown in Italy. J Genet Breed 43:17-24

32. Bagulho AS, Muacho MC, Carrillo JM, Brites C (2003) Influence of glutenin and puroindoline composition on the quality of bread wheat varieties grown in Portugal. In: Lafiandra D, Masci S, D'Ovidio R (eds) The gluten Proteins, Proceedings of the 8th international gluten workshop, Universita `degli Studi della Tuscia',Viterbo, Italy. The Royal Society of Chemistry, Cambridge, UK, pp 113-116
33. Branlard G, Dardevet M (1985) Diversity of grain proteins and bread wheat quality: i. Correlation between gliadin bands and flour quality characteristics. J Cereal Sci 3:329-343

34. Van Lonkhuijsen HJ, Hamer RJ, Schreuder C (1992) Influence of specific gliadins on the breadmaking quality of wheat. Cereal Chem 69:174-177

35. Khatkar BS, Fido RJ, Tatham AS, Schofield JD (2002) Functional properties of wheat gliadins. II. Effects on dynamic rheological properties of wheat gluten. J Cereal Sci 3:307-313. https://doi. org/10.1006/jers.2001.0429

36. Kohajdová Z, Karovičová J (2008) Nutritional value and baking applications of spelt wheat. Acta Sci Pol Technol Aliment 7:5-14

37. Pruska Kedzior A, Kedzior Z, Klockiewicz Kaminska E (2008) Comparison of viscoelastic properties of gluten from spelt and common wheat. Eur Food Res Technol 227:199-207. https://doi. org/10.1007/s00217-007-0710-0

38. Labuschagne MT, Claassen A, Van Deventer CS (1997) Biscuitmaking quality of backcross derivates of wheat differing in kernel hardness. Euphytica 96:263-266

39. Branlard G, Dardevet M, Saccomano R, Lagoutte F, Gourdon J (2001) Genetic diversity of wheat storage proteins and bread wheat quality. Euphytica 119:59-67

40. Filipčev B, Śimurina O, Bodroža-Solarov M, Obreht D (2013) Comparison of the bread-making performance of spelt varieties grown under organic conditions in the environment of northern Serbia and their responses to dough strengthening improvers. Hem In 67:44453. https://doi.org/10.2298/HEMIND120606083F

41. McRitchie F (1987) Evaluation of contributions from wheat protein fractions to dough mixing and bread making. J Cereal Sci 6:259-268

42. Barak S, Mudgil D, Khatkar BS (2013) Relationship of gliadin and glutenin proteins with dough rheology, flour pasting and bread making performance of wheat varieties. LWT-Food Sci Technol 51:211-217. https://doi.org/10.1016/j.lwt.2012.09.011

Publisher's Note Springer Nature remains neutral with regard to jurisdictional claims in published maps and institutional affiliations. 